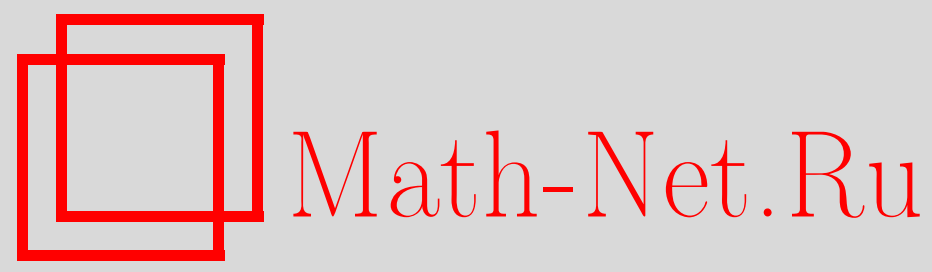

Л. Д. Менихес, О регуляризуемости некоторых классов отображений, обратных к интегральным операторам, $M a$ тем. заметки, 1999, том 65, выпуск 2, 222-229

DOI: https://doi.org/10.4213/mzm1044

Использование Общероссийского математического портала Math-Net.Ru подразумевает, что вы прочитали и согласны с пользовательским соглашением http://www.mathnet.ru/rus/agreement

Параметры загрузки:

IP : 3.85 .183 .62

26 апреля 2023 г., 09:28:20

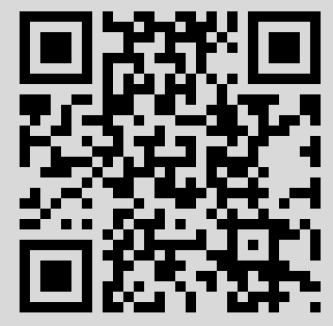




\section{О РЕГУЛЯРИЗУЕМОСТИ \\ НЕКОТОРЫХ КЛАССОВ ОТОБРАЖЕНИЙ, ОБРАТНЫХ К ИНТЕГРАЛЬНЫМ ОПЕРАТОРАМ}

\section{Л. Д. Менихес}

Исследуется регуляризуемость отображений, обратных к интегральным операторам, действуюшим из $C(0,1)$ в $L_{2}(0,1)$, в случае неинъективности продолжения операторов на $L_{2}(0,1)$. Построены классы таких операторов как с регуляризуемыми, так и с нерегуляризуемыми обратными отображениями, для которых продолжение операторов на $L_{2}(0,1)$ имеет бесконечномерное ядро.

Библиограффия: 6 названий.

Понятие регуляризуемости отображений, обратньх к линейным операторам, возникло при приближенном решении уравнений. Если такое отображение регуляризуемо, то уравнение может быть приближенно решено методом регуляризации А.Н. Тихонова. Если же отображение нерегуляризуемо, то метод Тихонова для приближенного решения уравнения с таким оператором не применим. Большой интерес представляет исследование вопроса о регуляризуемости отображений, обратных к интегральным операторам, так как оно связано с возможностью решения интегральных уравнений. В работе [1] показано, что для интегрального оператора из $C(0,1)$ в $L_{2}(0,1)$ при условии инъективности его продолжения на пространство $L_{2}(0,1)$ обратное отображение регуляризуемо, а в работе [2] построен интегральный оператор из $C(0,1)$ в $L_{2}(0,1)$ с нерегуляризуемым обратным отображением. В работах [3], [4] доказано существование интегральных операторов с нерегуляризуемыми обратньми в различных функциональных пространствах.

В данной работе исследуется связь регуляризуемости обратных для интегральных операторов из $C(0,1)$ в $L_{2}(0,1)$ со структурой ядра продолженного на $L_{2}(0,1)$ оператора. Приводится конструкция, позволяющая строить классы операторов как с регуляризуемыми обратными, так и классы операторов с нерегуляризуемыми обратными отображениями.

1. Пусть $X$ и $Y$ - банаховы пространства, $A: X \rightarrow Y$ - непрерывный линейньй инъективньй оператор. Мы будем говорить, что отображение $A^{-1}$ регуляризуемо, если существует семейство отображений $R_{\delta}: Y \rightarrow X, \delta \in\left(0, \delta_{0}\right)$, такое, что для любого $x \in X$

$$
\lim _{\delta \rightarrow 0} \sup _{\|y-A x\| \leqslant \delta}\left\|R_{\delta} y-x\right\|=0 .
$$

Хорошо известно (см. [5]), что если $X$ - квазирефлексивное (в частности, рефлексивное) пространство, то отображение $A^{-1}$ регуляризуемо для любого $A$. Поэтому если интегральньй оператор действует из $L_{2}(0,1)$ в $L_{2}(0,1)$, то обратньй оператор всегда 
является регуляризуемым. Совсем иная картина наблюдается при рассмотрении интегральных операторов из $C(0,1)$ в $L_{2}(0,1)$. Здесь существуют интегральные операторы с нерегуляризуемым обратным отображением. Напомним (см. [5]), что регуляризуемость отображения $A^{-1}$ эквивалентна необращению в 0 характеристики подпространства $A^{*} Y^{*} \subset X^{*}$.

Довольно сильное достаточное условие регуляризуемости состоит в следующем [6]. Если $E_{1}, E_{2}, E_{3}$ - банаховы пространства, $E_{1}$ сепарабельно, $E_{2}$ рефлексивно, $A: E_{1} \rightarrow E_{2}$ и $B: E_{2} \rightarrow E_{3}$ - линейные непрерьвные инъективные операторы и отображение $A^{-1}$ регуляризуемо, то регуляризуемо и обратное к произведению операторов отображение $(B A)^{-1}$.

Отсюда сразу следует уже упоминавшееся утверждение. Если продолжение по непрерьвности оператора $A: C(0,1) \rightarrow L_{2}(0,1)$ на все пространство $L_{2}(0,1)$ инъективно, то $A^{-1}$ регуляризуемо. Это следует из регуляризуемости $i^{-1}$, где $i: C(0,1) \rightarrow L_{2}(0,1)-$ вложение.

Если же указанное продолжение оператора $A$ имеет ненулевое ядро, то $A^{-1}$ может не быть регуляризуемым (см. [2]). В соответствующем примере из [2] ядро продолжения оператора $A$ было бесконечномерньм. Покажем сначала, что это не случайно, т.е. если это ядро конечномерно, то $A^{-1}$ регуляризуемо.

Теорема 1. Пусть $E_{1}, E_{2}, E_{3}$ - банаховы пространства, $E_{1}$ сепарабельно и $E_{2}$ рефлексивно, $A: E_{1} \rightarrow E_{2} u B: E_{2} \rightarrow E_{3}$ - линейные непрерывные операторы, $A$ инбективный оператор, $\operatorname{ker} B$ - конечномерное подпространство, $A^{-1}$ регуляризуемо и $A\left(E_{1}\right) \cap \operatorname{ker} B=\{0\}$, m.е. $B A-$ ингективный оператор. Тогда отображение $(B A)^{-1}$ регуляризуемо.

ДоказАтЕЛьСтво. Обозначим через $p$ естественноеотображение $p: E_{2} \rightarrow E_{2} / \operatorname{ker} B$. Тогда оператор $B$ можно представить в виде произведения $B=C p$, где $C$ - линейньй непрерьвньй инъективньй оператор, $C: E_{2} / \operatorname{ker} B \rightarrow E_{3}$. Ввиду рефлексивности факторпространства $E_{2} / \operatorname{ker} B$ и инъективности $C$

$$
\overline{C^{*} E_{3}^{*}}=\left(E_{2} / \operatorname{ker} B\right)^{*}
$$

Далее, так как сопряженное к факторпространству $E_{2} / \operatorname{ker} B$ есть аннулятор ядра ker $B$ и $\operatorname{ker} B$ конечномерно, то $p^{*}\left(E_{2} / \operatorname{ker} B\right)^{*}-$ подпространство конечной коразмерности в $E_{2}^{*}$.

Теперь из (1) следует

$$
\begin{aligned}
\overline{(B A)^{*} E_{3}^{*}} & =\overline{(C p A)^{*} E_{3}^{*}}=\overline{A^{*} p^{*} C^{*} E_{3}^{*}} \supset A^{*} \overline{p^{*} C^{*} E_{3}^{*}} \\
& \supset A^{*} p^{*} \overline{C^{*} E_{3}^{*}}=A^{*} p^{*}\left(E_{2} / \operatorname{ker} B\right)^{*}
\end{aligned}
$$

Регуляризуемость отображения $A^{-1}$ влечет ненулевую характеристику $A^{*} E_{2}^{*} \subset E_{1}^{*}$. Но из (2) следует, что подпространство $\overline{(B A)^{*} E_{3}^{*}} \subset E_{1}^{*}$ содержит образ при отображении $A^{*}$ подпространства конечной коразмерности в $E_{2}^{*}$, т.е. $\overline{(B A)^{*} E_{3}^{*}}$ содержит подпространство конечной коразмерности из $A^{*} E_{2}^{*}$. Так как тотальное подпространство конечного дефекта в пространстве ненулевой характеристики также имеет отличную от 0 характеристику, характеристика $\overline{(B A)^{*} E_{3}^{*}}$ отлична от 0. Следовательно, и $(B A)^{*} E_{3}^{*}$ имеет ненулевую характеристику, что влечет регуляризуемость отображения $(B A)^{-1}$. Теорема доказана. 
СлЕДСТВИЕ. Если интегральный оператор $A: C(0,1) \rightarrow L_{2}(0,1)$ инбективен $u$ его продолхение на $L_{2}(0,1)$ имеет конечномерное ядро, то $A^{-1}$ регуляризуемо.

Заметим, что условие конечномерности ядра $\operatorname{ker} B$ в теореме 1 не может быть снято, это следует из результатов работы [2].

2. Изучим более детально случай, когда $\operatorname{ker} B$ бесконечномерно. А именно, мы построим как классы операторов из $C(0,1)$ в $L_{2}(0,1)$, продолжение которых на $L_{2}(0,1)$ имеет бесконечномерное ядро, а их обратные регуляризуемы, так и классы операторов с аналогичным свойством, для которых обратные отображения нерегуляризуемы.

Сначала рассмотрим метод построения подпространств в $C^{*}(0,1)$ с различной характеристикой. Пусть дана последовательность положительных чисел $\left(a_{n}\right)$. Построим по этой последовательности некоторые последовательности функций, определенных на промежутке $[0,1]$. Для этого введем следующие последовательности промежутков:

$I_{k}^{-}=\left[\frac{2^{k}-1}{2^{k}}, \frac{2^{k+2}-3}{2^{k+2}}\right), I_{k}^{+}=\left[\frac{2^{k+2}-3}{2^{k+2}}, \frac{2^{k+1}-1}{2^{k+1}}\right], \quad I_{k}=I_{k}^{-} \cup I_{k}^{+}, \quad k=1,2, \ldots$,

и следующие последовательности функций: $\alpha_{k}(t)=0$ при $t \in[0,1] \backslash I_{k}^{+}, \alpha_{k}\left(\left(2^{k+3}-5\right) /\right.$ $\left.2^{k+3}\right)=a_{k}, \alpha_{k}(t)$ линейна на промежутках

$$
\left[\frac{2^{k+2}-3}{2^{k+2}}, \frac{2^{k+3}-5}{2^{k+3}}\right],\left[\frac{2^{k+3}-5}{2^{k+3}}, \frac{2^{k+1}-1}{2^{k+1}}\right]
$$

и непрерьвна на $[0,1] ; \beta_{k}(t)=0$ при $t \in[0,1] \backslash I_{k}^{-}$, линейна на $I_{k}^{-}$, непрерьвна при $t=\left(2^{k}-1\right) / 2^{k}$ и

$$
\lim _{t \rightarrow\left(2^{k+2}-3\right) / 2^{k+2}-0} \beta_{k}(t)=1 ;
$$

$\gamma_{k n}(t)=0$ при $t \in[0,1] \backslash I_{k}$,

$$
\gamma_{k n}(t)=-\beta_{k}(t) \quad \text { при } \quad t \in\left[\frac{2^{k}-1}{2^{k}}, \frac{2^{k+2}-3}{2^{k+2}}-\frac{\left|I_{k}^{-}\right|}{n}\right],
$$

где $\left|I_{k}^{-}\right|$- длина промежутка $I_{k}^{-}, k, n=1,2, \ldots, \gamma_{k n}\left(\left(2^{k+2}-3\right) / 2^{k+2}\right)=1, \gamma_{k n}\left(t_{k}\right)=$ $\alpha_{k}\left(t_{k}\right) / a_{k}$, где $t_{k}-$ меньший из двух корней уравнения

$$
\left(2+\frac{1}{a_{k}}\right) \alpha_{k}(t)=1
$$

$\gamma_{k n}(t)=\alpha_{k}(t) / a_{k}$ при $t \in\left[t_{k},\left(2^{k+1}-1\right) / 2^{k+1}\right]$, непрерывна на $[0,1]$ и линейна на промежутках

$$
\left[\frac{2^{k+2}-3}{2^{k+2}}-\frac{\left|I_{k}^{-}\right|}{n}, \frac{2^{k+2}-3}{2^{k+2}}\right],\left[\frac{2^{k+2}-3}{2^{k+2}}, t_{k}\right] .
$$

Отметим, что функции $\alpha_{k}(t)$ и $\gamma_{k n}(t)$ непрерьвны на $[0,1],\left|\gamma_{k n}(t)\right| \leqslant 1$ при $t \in[0,1]$, $\beta_{k}(t)$ разрьвна на $[0,1]$ и $\left|\beta_{k}(t)\right| \leqslant 1$ при $t \in[0,1]$.

Пусть $\delta_{k}(t)=\alpha_{k}(t)+\beta_{k}(t), k=1,2, \ldots$. Обозначим через $M \subset L_{2}(0,1)$ замкнутое подпространство в $L_{2}(0,1)$, натянутое на $\left(\delta_{k}(t)\right)$, и через $p$ естественное отображение $p: L_{2}(0,1) \rightarrow L_{2}(0,1) / M$ на факторпространство. Будем рассматривать подпространства в $C^{*}(0,1)$, которые являются образом сопряженного оператора $(p i)^{*}$, где 
$i: C(0,1) \rightarrow L_{2}(0,1)$ - вложение. Так как в $M$ все функции, кроме тождественного нуля, разрьвны, то $p i$ - инъективньй оператор и, следовательно, $(p i)^{*}\left(L_{2}(0,1) / M\right)^{*} \subset$ $C^{*}(0,1)$ - тотальное подпространство. В теореме 2 мы вычислим характеристику этого подпространства.

Напомним способ вычисления характеристики подпространств. Если $A: X \rightarrow Y-$ линейньй непрерьвньй инъективный оператор, то характеристика $r\left(A^{*} Y^{*}\right)$ подпространства $A^{*} Y^{*} \subset X^{*}$ может быть найдена как обратная величина верхней грани чисел $\|x\|$, где $x$ пробегает замыкание единичного шара $S$ в $X$ по новой норме $\|x\|_{1}=\|A x\|$. Следовательно, для нахождения характеристики $r\left((p i)^{*}\left(L_{2}(0,1) / M\right)^{*}\right)$ достаточно вычислить верхнюю грань $\|x\|_{C}$, где $x$ пробегает замькание единичного шара в $C(0,1)$ по норме $\|x\|_{1}=\|p i x\|$ и в правой части стоит норма в $L_{2}(0,1) / M$.

ЛЕмма 1. Если непрерывная функиия $x(t)$ принадлежит замыканию единичного шара $S_{1}$ в $C(0,1)$ по норме $\|\cdot\|_{1}$, то для любой точки разрыва $t_{0}$ функиий из $M$ ( такие точки пробегают счетное множество середин отрезков $I_{k}$ ) выполняется соотношение $\left|x\left(t_{0}\right)\right| \leqslant 1$.

ДокАЗАТЕЛьСТво. Проведем доказательство для случая $x\left(t_{0}\right) \geqslant 0$ (если $x\left(t_{0}\right)<0$, то рассуждения аналогичны). Докажем, что $x\left(t_{0}\right) \leqslant 1$. Пусть, напротив, $x\left(t_{0}\right)>1$. Тогда в силу непрерывности $x(t)$ сушествуют числа $\alpha>0$ и $\delta>0$ такие, что при $\left|t-t_{0}\right|<\delta$ вьполняется неравенство

$$
x(t)>1+\alpha
$$

По условию существует последовательность непрерьвных функций $\left(x_{n}(t)\right),\left|x_{n}(t)\right| \leqslant 1$, такая, что $\left\|x_{n}-x\right\|_{1} \rightarrow 0$ при $n \rightarrow \infty$. Это означает, что существует последовательность $\omega_{n}(t) \in M$ такая, что $\left\|x_{n}-x-\omega_{n}\right\| \rightarrow 0$ по $L_{2}$-норме. Пусть $t_{0}$ является серединой $I_{k}$. Тогда сужение функции $\omega_{n}$ на $I_{k}^{+}$имеет вид

$$
\left.\omega_{n}\right|_{I_{k}^{+}}=C_{n} \alpha_{k}(t)
$$

Изучим поведение последовательности $\left(C_{n}\right)$. Во-первых, $\left(C_{n}\right)$ не может быть ограниченной последовательностью. В противном случае из (3) следует, что при $\left|t-t_{0}\right|<\delta$ и $t \in I_{k}$

$$
x(t)-x_{n}(t)>\alpha
$$

Из (4) и (5) вытекает существование малого $\delta_{1}$ такого, что при $t \in\left(t_{0}, t_{0}+\delta_{1}\right)$ разность $x(t)-x_{n}(t)-\omega_{n}(t)$ превышает некоторую фиксированную константу, что противоречит сходимости $\left\|x_{n}-x-\omega_{n}\right\| \rightarrow 0$. Во-вторых, последовательность $\left(C_{n}\right)$ не может быть неограниченной. В самом деле, функция $x(t)$ непрерывна, значит ограничена на $[0,1]$. При условии неограниченности $\left(C_{n}\right)$ из (4) следует, что разность $\left|x(t)-x_{n}(t)-\omega_{n}(t)\right|$ на множестве положительной меры из $I_{k}^{+}$превышает некоторую константу для бесконечного числа номеров $n$. А это противоречит сходимости $\left\|x_{n}-x-\omega\right\| \rightarrow 0$.

Итак, последовательность $\left(C_{n}\right)$ не может быть ни ограниченной, ни неограниченной. Полученное противоречие доказьвает лемму. 
Tеорема 2. Характеристика подпространства $(\text { pi })^{*}\left(L_{2}(0,1) / M\right)^{*} \subset C^{*}(0,1)$ находится следуюшим образом:

$$
r\left((p i)^{*}\left(L_{2}(0,1) / M\right)^{*}\right)= \begin{cases}\frac{1}{3} & \text { при } a \in(0,1], \\ \frac{1}{2 a+1} & \text { nри } a \in[1, \infty), \quad a=\sup a_{n} . \\ 0 & \text { при } a=\infty,\end{cases}
$$

ДокАЗАТЕЛЬСтво. Докажем равносильное утверждение:

$$
\sup \left\{\|x\|_{C}: x \in \bar{S}_{1}\right\}= \begin{cases}3 & \text { при } a \in(0,1], \\ 2 a+1 & \text { при } a \in[1, \infty), \\ \infty & \text { при } a=\infty\end{cases}
$$

Здесь $\bar{S}_{1}$ - замыкание единичного шара в $C(0,1)$ по норме $\|x\|_{1}=\|p i x\|_{L_{2} / M}$.

Рассмотрим последовательность непрерывных функций $\left(x_{k}(t)\right)$, определенную следуюшим образом:

$$
x_{k}(t)= \begin{cases}\beta_{k}(t) & \text { при } t \in I_{k}^{-}, \\ 1 & \text { при } t \in I_{k}^{+} \cap\left(-\infty, t_{0}\right], \\ \left(2+\frac{1}{a_{k}}\right) \alpha_{k}(t) & \text { при } t \in I_{k}^{+} \cap\left[t_{0}, \infty\right), \\ 0 & \text { при } t \in[0,1] \backslash I_{k} .\end{cases}
$$

Ясно, что $\sup \left\{x_{k}(t): t \in[0,1]\right\}=2 a_{k}+1$, т.е. $\left\|x_{k}\right\|_{C}=2 a_{k}+1$. Покажем, что $x_{k}(t) \in \bar{S}_{1}$ для любого $k=1,2, \ldots$. Для этого проверим, что $\gamma_{k n}(t) \rightarrow x_{k}(t)$ при $n \rightarrow \infty$ для любого $k$ по норме $\|\cdot\|_{1}$. По построению $\gamma_{k n}$ мера

$$
\mu\left\{t: x_{k}(t)-\gamma_{k n}(t) \neq 2 \delta_{k}(t)\right\}=\frac{\left|I_{k}^{-}\right|}{n} .
$$

Принимая во внимание ограниченность при фиксированном $k$ функций $x_{k}(t), \gamma_{k n}(t)$, $\delta_{k}(t)$, из $(7)$ выводим сходимость $x_{k}(t)-\gamma_{k n}(t)-2 \delta_{k}(t) \rightarrow 0$ при $n \rightarrow \infty$ в $L_{2}(0,1)$. Так как $\delta_{k}(t) \in M$ при всех $k$, имеем сходимость $\gamma_{k n}(t) \rightarrow x_{k}(t)$ при $n \rightarrow \infty$ по норме $\|\cdot\|_{1}$, т.е. $x_{k}(t) \in \bar{S}_{1}$. Таким образом, мы показали, что

$$
\sup \left\{\|x\|_{C}: x \in \bar{S}_{1}\right\} \geqslant 2 a+1 .
$$

Отсюда сразу следует последнее из равенств (6).

Теперь покажем, что вьполнено второе из равенств (6). Итак, пусть $a \geqslant 1$. Вследствие (8) достаточно показать, что

$$
\sup \left\{\|x\|_{C}: x \in \bar{S}_{1}\right\} \leqslant 2 a+1 .
$$

Пусть, напротив, $\sup \left\{\|x\|_{C}: x \in \bar{S}_{1}\right\}>2 a+1$. Это означает, что найдется непрерывная функция $x(t) \in \bar{S}_{1}$ и $\|x\|_{C}>2 a+1$. Пусть $t^{*} \in[0,1]$ таково, что $x\left(t^{*}\right)>2 a+1$ (если $x\left(t^{*}\right)<0$, то рассуждения аналогичны). Тогда найдутся $\alpha>0$ и $\delta>0$ такие, что

$$
x(t)>2 a+1+\alpha
$$


при $\left|t-t^{*}\right|<\delta$.

Существуют последовательности $\omega_{n} \in M$ и $z_{n} \in S_{1}$ в $C(0,1)$ такие, что

$$
\left\|x-z_{n}-\omega_{n}\right\| \rightarrow 0
$$

в пространстве $L_{2}(0,1)$. Из $(10)$ следует, что $x(t)-z_{n}(t)>2 a+\alpha$ при $\left|t-t^{*}\right|<\delta$, а из $(11)$ - что найдется такой номер $k_{0}$, для которого коэффищиент $C_{n}$ из $(4)$, начиная с некоторого номера $n=N$, удовлетворяет неравенству

$$
C_{n}>2+\alpha_{1}
$$

$\alpha_{1}>0$ - некоторое число (здесь мы использовали условие $a \geqslant 1$ ). Обозначим через $t_{0}$ середину отрезка $I_{k_{0}}$. В силу леммы $1 x\left(t_{0}\right) \leqslant 1$, значит в некоторой окрестности $t_{0}$ выполняется неравенство $x(t)<1+\alpha_{1} / 2$, из которого следует

$$
x(t)-z_{n}(t)<2+\frac{\alpha_{1}}{2} .
$$

Из (12) и (13) следует, что в некоторой левой полуокрестности точки $t_{0}$ разность $x(t)-z_{n}(t)-\omega_{n}(t)$ превышает некоторую фиксированную константу, что противоречит соотношению (11). Итак, доказано соотношение (9), что вместе с (8) влечет выполнимость второго из равенств (6). Осталось доказать первое из равенств (6).

Пусть $a \in(0,1]$. Покажем, что

$$
\sup \left\{\|x\|_{C}: x \in \bar{S}_{1}\right\} \geqslant 3 .
$$

Введем следующую последовательность функций: $\xi_{n}(t)=0$ при $t \in[0,1] \backslash[(5 n-2) /$ $(8 n),(5 n-1) /(8 n)], \xi_{n}((10 n-3) /(16 n))=\beta_{1}((10 n-3) /(16 n))$, непрерывна на $[0,1]$ и линейна на промежутках

$$
\left[\frac{5 n-2}{8 n}, \frac{10 n-3}{16 n}\right],\left[\frac{10 n-3}{16 n}, \frac{5 n-1}{8 n}\right] .
$$

Пусть $y_{m}(t)=x_{1}(t)+2 \xi_{m}(t), m=2,3, \ldots$ Покажем, что $y_{m} \in \bar{S}_{1}$. Действительно, вышемы показали, что $\gamma_{k n}(t) \rightarrow x_{k}(t)$ при $n \rightarrow \infty$ по норме $\|\cdot\|_{1}$ для любого $k$. Возьмем $k=1$. Тогда $\gamma_{1 n}(t) \rightarrow x_{1}(t)$ или $\gamma_{1 n}(t)+2 \xi_{m}(t) \rightarrow y_{m}(t)$ для любого $m=2,3, \ldots$ Осталось показать, что

$$
\gamma_{1 n}(t)+2 \xi_{m}(t) \in S_{1}
$$

Для произвольных $m$ и $n$ это не так, но непосредственно из построения функций видно, что при $n \geqslant m$ соотношение (15) вьполняется. Этого достаточно для доказательства того, что $y_{m} \in \bar{S}_{1}$. В силу построения функций имеем $\sup \left\{\left\|y_{m}\right\|_{C}: m=2,3, \ldots\right\}=3$. Тем самьм, соотношение (14) доказано.

Покажем, что верно и обратное неравенство

$$
\sup \left\{\|x\|_{C}: x \in \bar{S}_{1}\right\} \leqslant 3
$$

Неравенство (16) доказывается точно так же, как и (9) при $a=1$. Тот факт, что в нашем случае $a \leqslant 1$, не влияет на доказательство. Таким образом, соотношения (14) и (16) доказьвают первое из равенств (6). Теорема доказана. 
СлЕДСТВИЕ. Если $\sup a_{n}<\infty$, то отображсение $(p i)^{-1}$ регуляризуемо; если $\sup a_{n}=\infty$, mо $(p i)^{-1}$ нерегуляризуемо.

Заметим, что в любом случае $\operatorname{ker} p=M$ бесконечномерно, так что теорема 1 здесь не применима. Новым является утверждение о том, что и в случае бесконечномерности ядра $p$, обратное отображение к произведению $p i$ может быть регуляризуемьм.

3. Теперь применим изложенную выше теорию для изучения регуляризуемости отображений, обратных к интегральным операторам, действующим из $C(0,1)$ в $L_{2}(0,1)$.

Будем рассматривать интегральные операторы с гладкими ядрами, хотя вся теория проходит и для непрерывных ядер. Пусть $K(x, t)$ - бесконечно дифференцируемая функция на квадрате $[0,1] \times[0,1]$.

Рассмотрим интегральньй оператор

$$
Q: f(x) \mapsto \int_{0}^{1} K(x, t) f(t) d t
$$

действующий из $C(0,1)$ в $L_{2}(0,1)$, и интегральный оператор

$$
Q^{\prime}: f(x) \mapsto \int_{0}^{1} K(x, t) f(t) d t
$$

действующий из $L_{2}(0,1)$ в $L_{2}(0,1)$.

Сложность изучения регуляризуемости $Q^{-1}$, в частности, состоит в том, что и в случае инъективности оператора $Q$ оператор $Q^{\prime}$ может не быть инъективным и даже иметь бесконечномерноеядро. Основная наша идея состоит в том, что можно построить интегральные операторы, инъективные из $C(0,1)$ в $L_{2}(0,1)$, продолжение которых на $L_{2}(0,1)$ имеет в качестве ядра построенные вьше подпространства $M$.

Лемма 2. Пусть $M$ - любое из построенных выше подпространств в $L_{2}(0,1)$. Тогда в ортогональном дополнении $N \kappa M$ существует полная ортонормированная система $\left\{\psi_{n}(t)\right\}$ такая, что $\psi_{n}(t) \in C_{0}^{\infty}(0,1), n=1,2, \ldots$.

Лемма доказьвается так же, как и аналогичное утверждение в [2], только для несколько иного $M$.

Теперь, как и в [2], рассмотрим интегральные операторы $Q$ и $Q^{\prime}$ с ядром

$$
K(x, t)=\sum_{n=1}^{\infty} a_{n} \varphi_{n}(x) \psi_{n}(t)
$$

где $\left\{\varphi_{n}(x)\right\}$ - произвольная ортонормированная система, состоящая из бесконечно дифференцируемых на отрезке $[0,1]$ функций, и

$$
a_{n}=\left(\sup _{0 \leqslant x \leqslant 1} \max \left\{\left|\varphi_{n}(x)\right|, \ldots,\left|\varphi_{n}^{(n)}(x)\right|\right\}\right)^{-1}\left(\sup _{0 \leqslant t \leqslant 1} \max \left\{\left|\psi_{n}(t)\right|, \ldots,\left|\psi_{n}^{(n)}(t)\right|\right\} n^{2}\right)^{-1}
$$

Ясно, что $K(x, t)$ - бесконечно дифференцируемая функция на квадрате $[0,1] \times[0,1]$.

ТЕОРема 3. Пусть $Q$-интегральный оператор, построенный по формулам (17), (18). Тогда $Q$ - ингективный оператор, и если $\sup a_{n}<\infty$, то отображение $Q^{-1}$ регуляризуемо, а если $\sup a_{n}=\infty$, то $Q^{-1}$ нерегуляризуемо. 
ДокАЗАТЕльство. Сначала покажем, что $\operatorname{ker} Q^{\prime}=M$. Действительно, если

$$
\int_{0}^{1}\left(\sum_{n=1}^{\infty} a_{n} \varphi_{n}(x) \psi_{n}(t) f(t)\right) d t=0
$$

TO

$$
\sum_{n=1}^{\infty} a_{n} b_{n} \varphi_{n}(x)=0
$$

где $b_{n}=\int_{0}^{1} f(t) \psi_{n}(t) d t-n$-й коэффициент Фурье функции $f(t)$ по системе $\left\{\psi_{n}(t)\right\}$, так как ряд в (19) по теореме Лебега можно почленно интегрировать. Из (20) следует $b_{n}=0, n=1,2, \ldots$, т.е. $f(t) \in M$. Ясно также, что из $f(t) \in M$ следует $f(t) \in \operatorname{ker} Q^{\prime}$. Таким образом, доказано, что $\operatorname{ker} Q^{\prime}=M$. Отсюда следует, что, во-первых, $Q$ - инъективньй оператор и, во-вторых, $Q$ можно представить в виде произведения операторов

$$
Q=Q^{\prime} i=u p i
$$

где $u: L_{2}(0,1) / M \rightarrow L_{2}(0,1)$ - инъективньй оператор.

Пусть $\sup a_{n}=\infty$. Тогда по теореме $2(p i)^{-1}$ нерегуляризуемо. Значит, подпространство $(p i) *\left(L_{2}(0,1) / M\right)^{*}$ имеет нулевую характеристику, но из (21) следует, что $Q^{*} L_{2}^{*}(0,1)=(p i)^{*} u^{*} L_{2}^{*}(0,1) \subset(p i)^{*}\left(L_{2}(0,1) / M\right)^{*}$, т.е. $Q^{*} L_{2}^{*}(0,1)$, тем более, имеет нулевую характеристику, что влечет нерегуляризуемость отображения $Q^{-1}$.

Пусть теперь $\sup a_{n}<\infty$. Тогда по теореме $2(p i)^{-1}$ регуляризуемо, т.е. подпространство $(p i)^{*}\left(L_{2}(0,1) / M\right)^{*}$ имеет ненулевую характеристику. Далее, ввиду рефлексивности $L_{2}(0,1) / M$ и инъективности оператора $u \overline{u^{*} L_{2}^{*}(0,1)}=\left(L_{2}(0,1) / M\right)^{*}$ и, следовательно,

$$
\overline{Q^{*} L_{2}^{*}(0,1)} \supset(p i)^{*} \overline{u^{*} L_{2}(0,1)}=(p i)^{*}\left(L_{2}(0,1) / M\right)^{*} .
$$

Это означает, что $\overline{Q^{*} L_{2}^{*}(0,1)}$, стало быть, и $Q^{*} L_{2}^{*}(0,1)$ - подпространства ненулевой характеристики, что влечет регуляризуемость $Q^{-1}$. Теорема доказана.

Можно считать, что оператор $Q$ имеет симметричное ядро. Для этого достаточно положить $\varphi_{n}(x)=\psi_{n}(x), n=1,2, \ldots$.

Таким образом, найден класс интегральных операторов из $C(0,1)$ в $L_{2}(0,1)$, для которых обратные отображения регуляризуемы, и класс интегральных операторов из $C(0,1)$ в $L_{2}(0,1)$, для которых обратные отображения нерегуляризуемы. В обоих случаях продолжение операторов на $L_{2}(0,1)$ имеет бесконечномерное ядро.

\section{СПИСОК ЦИТИРОВАННОЙ ЛИТЕРАТУРЫ}

[1] Танана В. П. О решении интегральных уравнений Фредгольма первого рода в пространстве $C(0,1) / /$ Матем. записки. Т. 7. Тетрадь 4. Свердловск: УрГУ, 1970. С. 83-90.

[2] Менихес Л. Д. О регуляризуемости отображений, обратных к интегральным операторам // Докл. АН СССР. 1978. Т. 241. № 2. С. 282-285.

[3] Пличко А.Н. Ненормирующие подпространства и интегральные операторы с нерегуляризуемым обратным // Сиб. матем. ж. 1988. Т. 29. № 4. С. 208-211.

[4] Островский М.И. Замечание об аналитической представимости отображений, обратных к интегральным операторам // Матем. физика, анализ, геометрия. 1994. Т. 1. № 3-4. С. 513-515.

[5] Винокуров В. А., Петунин Ю. И., Пличко А. Н. Условия измеримости и регуляризуемости отображений, обратных к непрерывным линейным отображениям // Докл. АН СССР. 1975. T. 220. № 3. C. 509-511.

[6] Петунин Ю.И., Пличко А. Н. Регуляризуемость по Тихонову некоторых классов некорректных задач // Матем. сборник. Киев: Наукова думка, 1976. С. 221-224. 